\title{
Limits of Tomographic Reconstruction of Discrete Nanoparticles
}

\author{
Xiongyao Wang ${ }^{\bullet}$, Marek Malac $^{\bullet}, \square$, Al Meldrum ${ }^{\bullet}$, Ross Lockwood ${ }^{\bullet}$, , Peng Li ${ }^{\bullet}$, \\ Hiromitsu Furukawa ${ }^{\circ}$ \\ - National Institute for Nanotechnology, Edmonton, 11421 Saskatchewan Drive, Canada. \\ ${ }^{\square}$ Department of Physics, University of Alberta, Edmonton, Canada. \\ oJEOL Systems Technology Co., Ltd. Akishima, Japan.
}

We discuss the limitations and practical issues of various tomography reconstruction methods applied to samples containing many discrete nanoparticles.

Electron tomography (ET) is widely used to study complex three-dimensional (3D) nanoscale structures. The 3D information is obtained from reconstruction of tilt series of projected images. Ideally the tilt series encompasses a full angular range $(\alpha=$ $\left.\pm 90^{\circ}\right)$; however in practice the tilt range is limited to a smaller value $\left( \pm \alpha<60^{\circ}\right)$ due to the geometrical constraints of the microscope, holder, microscope depth of focus and the sample itself. We investigate the image artifacts present in the $3 \mathrm{D}$ reconstruction of nanoparticles distributed in amorphous matrix, such as $\mathrm{Si}$ and $\mathrm{Er}$ in $\mathrm{SiOx}$ matrix [1], when the angular series is incomplete. The image artifacts arising from different tomographic reconstruction methods on a sample with a continuous boundary was discussed previously [2], but the case of multiple overlapping particles, although common in many nanomaterials systems, remains challenging.

To investigate limits of the reconstruction methods themselves a series of ghost data were generated by projecting a volume of randomly distributed discrete particles numerically generated in Matlab ${ }^{\mathrm{TM}}$, shown in Fig. 1, over $\pm 90^{\circ}$ tilt range in $1^{\circ}$ intervals. The use of computer-generated ghost data reduces difficulties related to image alignment, limited signal to noise ratio and tilt range. The objects in the ghost data had hard boundaries (digital value 1 inside objects and 0 outside) as were the computer-generated projections. The hard-boundary data correspond to an extreme case of detector saturation in scanning TEM tomography, a difficult situation for reconstruction. Two reconstruction methods were tested: Filtered Back-Projection (FBP) and Simultaneous Iterative Reconstruction Technique (SIRT), both implemented in TEMography ${ }^{\mathrm{TM}}$ package [3].

The results show the following: i). Two nearby particles marked in red in Fig.1 (a) are not separated either in FBP or SIRT reconstructions (50 iterations) as shown in Fig.2 (a). Our numerical results indicate that the centre-centre distance of two spheres must be larger than about 2.5 times its radius in order to be imaged as clearly separate. ii). The apparent elongation factor $e=\sqrt{\frac{\alpha+\sin \alpha \cos \alpha}{\alpha-\sin \alpha \cos \alpha}}$, where $\alpha$ is the tilt range, shows that a $\pm 90^{\circ}$ tilt angle should eliminate the elongation effect in [4]. However, the shape distortion is still observed in individual particles in a hard-boundary many-particle system as shown in Fig. $2 \mathrm{~b}$ even with range $\pm 90^{\circ}$ and $1^{\circ}$ step.

Fig.2 (b) shows a cross section through a SIRT reconstructed volume. Clearly particles did not regain their original spherical shape. The particle shape distortions appear to increase with decreasing distance between individual particles. It appears that compared to $\pm 70^{\circ}$ tilt range, a $\pm 90^{\circ}$ range does not provide a significant benefit in determining particle shape. 
Our results show that even on computer-generated projections from a computergenerated ghost sample quantitative measurement of shape and volume of sample with randomly distributed particles appears to be determined by the intrinsic limits of the (FBP and SIRT) reconstruction process itself. Of particular concern is the missing angular range (when the maximum tilt is less than about $70^{\circ}$ ), the sampling of particles in real space (the number of pixels per particle) and, the greyscale sampling (i.e. avoiding image saturation).

\section{References:}

[1] Peng Li, Xiongyao Wang, Marek Malac, Ray Egerton, Al Meldrum, X. Liang, F. Lenz, Carol J. Wang, Microscopy \& Microanalysis, Vol 15, Suppl.2, p.1256, (2009).

[2] H. Jinnai, R.J. Spontak, Polymer. 50 (2009) 1067.

[3] Temography.com

[4] P.A. Midgley and M. Weyland, Ultramicroscopy. 96 (2003) 413.

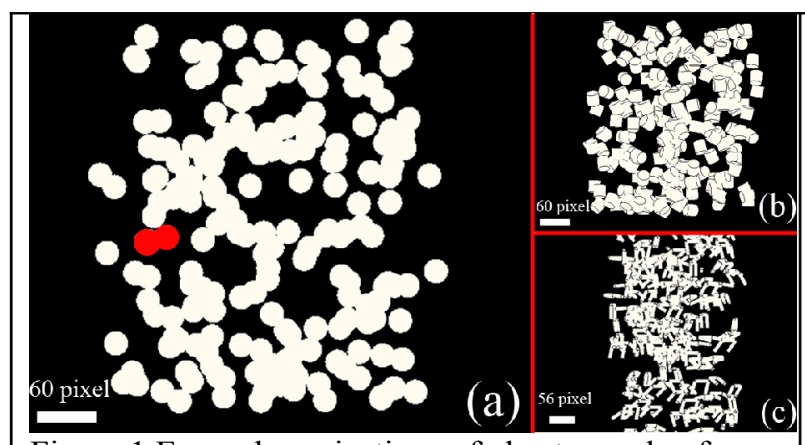

Figure 1 Example projections of ghost sample of randomly-distributed particles with different morphologies a) sphere b) cylinder c) horseshoe
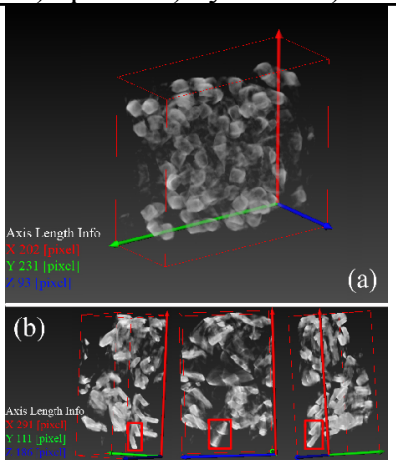

Figure 3 a) the result of reconstruction by SIRT ( 20 iterations) for cylindrical particles shows that the distortion effect is not obvious b) the red box shows the same horseshoe particle. The elongation effect is very obvious in the middle panel.
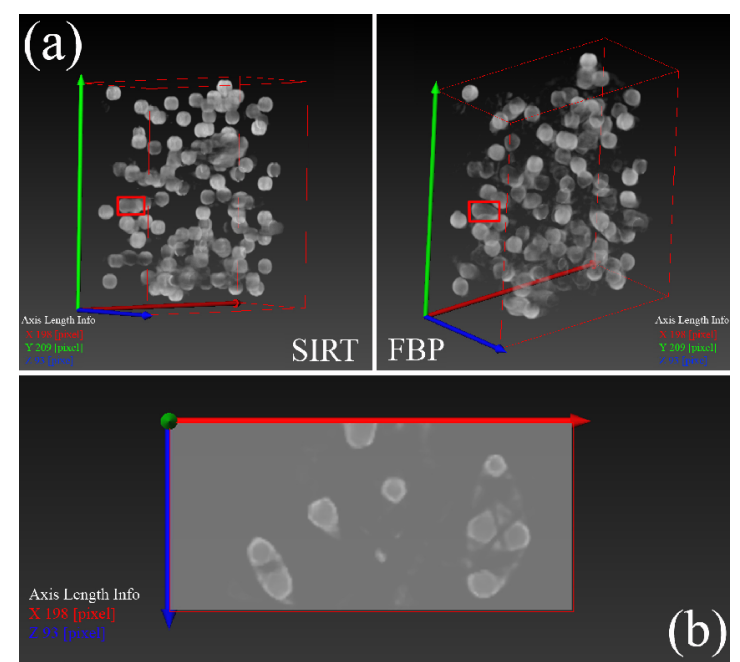

Figure 2 the result of $3 \mathrm{D}$ reconstruction for $\pm 90^{\circ}$ range tilt angle with $1^{\circ}$ step by SIRT (20 iterations) and FBP respectively. The red box shows that the two particles marked in red in Fig. 1 are not separated. b) the cross-section (Y axis view) of the reconstruction result (SIRT). 